\title{
Antibiotic resistance surveillance and control in the Mediterranean region: report of the ARMed Consensus Conference
}

\author{
Michael A. Borg ${ }^{1}$, Barry D. Cookson ${ }^{2}$, Peter Zarb ${ }^{1}$, Elizabeth A. Scicluna ${ }^{1}$, on behalf of the ARMed \\ Steering Group and Collaborators \\ ${ }^{1}$ Infection Control Unit, Mater Dei Hospital, Msida, Malta \\ ${ }^{2}$ Laboratory of Healthcare Associated Infection, Centre for Infections, Health Protection Agency, 61 Colindale Avenue, London, \\ United Kingdom
}

\begin{abstract}
Antimicrobial resistance has become a global threat to effective health care delivery. This is particularly the case within the Mediterranean region, where data from recent studies suggests the situation to be particularly acute. A better knowledge base, as well as a collaborative effort, is therefore required to address this ever increasing challenge to effective patient care. Over its four-year period, the Antibiotic Resistance Surveillance and Control in the Mediterranean Region (ARMed) project investigated the epidemiology of antimicrobial resistance, as well as its contributory factors, in a number of countries in the southern and eastern Mediterranean region through the collection of comparable and validated data. The project culminated in a consensus conference held in Malta in November 2006. The conference provided a forum for expert delegates to agree on a number of priority strategic recommendations that would be relevant to resistance containment efforts in the region. There was general agreement on the need for surveillance and audit to underpin any intervention to tackle antimicrobial resistance, both to monitor changing epidemiological trends in critical pathogens as well as to identify antibiotic consumption practices and effectiveness of prevention and control of health care associated infections. In addition, the importance to convey these data to key users was also stressed in all workshops, as was better education and training of health care workers. The recommendations also made it clear that ownership of the problem needs to be improved throughout the region and that resources, both financial as well as human, must be allocated by the respective policy makers in order to combat it.
\end{abstract}

\section{Keywords:}

J Infect Dev Ctries 2009; 3(9):654-659.

Received June 19, 2009 - Accepted August 03, 2009

Copyright (C) 2009 Borg et al. This is an open-access article distributed under the Creative Commons Attribution License, which permits unrestricted use, distribution, and reproduction in any medium, provided the original work is properly cited.

\section{Introduction}

Antimicrobial resistance is increasing and brings with it the possibility of untreatable infections and a return to the pre-antibiotic era. This situation impinges on the quality of patient care through its associated mortality, morbidity, and significant economic consequences [1].

The threat of antimicrobial resistance has been identified as one of the major challenges facing public health by numerous organizations and scientific societies including the World Health Organization (WHO) and the European Society for Clinical Microbiology and Infectious Diseases (ESCMID) [2]. They have emphasised the vital need to implement surveillance and intervention programmes on antimicrobial resistance to reduce the associated mortality, morbidity, and socio-economic costs. European Union (EU) Resolution 1999/C 195/01, "A strategy against the microbial threat", specifies research work on antimicrobial resistance as a priority and sets out the following as important initiatives:

- surveillance of antibiotic resistance in human medicine

- improved infection control both in hospitals and in non-hospital care

- promotion of optimal prescribing and use of antibiotics [3]

In the past, data about the prevalence of antimicrobial resistance and its drivers within the non-European countries of the Mediterranean has been lacking [4]. The Antibiotic Resistance Surveillance and Control in the Mediterranean Region (ARMed) project commenced on January 1, 2003, and was funded by the European Commission under the INCO-MED FP5 programme (ICA3-CT2002-10015) [5]. The project established a network of hospitals and laboratories in nine countries: 
Turkey, Tunisia, Egypt, Jordan, Morocco, Cyprus, Malta, Algeria, and Lebanon. Over its four-year span, it investigated antimicrobial resistance in these southern and eastern Mediterranean countries through the collection of comparable and validated resistance data as well as information about antibiotic consumption patterns and infection control initiatives.

The ARMed Consensus Conference marked the conclusion of this Thematic Network project and provided a first-time opportunity for stakeholders in the Euro-Mediterranean zone to share experiences over an issue that will need cooperation between all regional entities. At the same time, it also created the right ambience for reflective discussion to inform possible solutions relevant to the region and allow them to be put forward for action. The conference was held in Qawra, Malta, between the November 10 and 12, 2006, and was co-hosted by the European Commission, the World Health Organization, and ESCMID.

The Conference aimed to

- deliver a number of plenary symposia to provide an overview of the key ARMed results

- allow participants to discuss the ARMed data in the context of the whole Mediterranean region

- provide a forum for delegates to further explore the data presented within special interest workshops

- agree on a number of priority strategic recommendations that would be relevant to resistance containment efforts in the region

This report presents the Consensus Recommendations formulated by the participants of the three ARMed workshops from which proposals were adopted on the final day of the conference.

\section{Workshop 1: Surveillance of antibiotic resistance}

The project collected susceptibility test results from invasive strains of Staphylococcus aureus, Streptococcus pneumoniae, Escherichia coli, Enterococcus faecium, and faecalis routinely isolated from clinical samples of blood and cerebrospinal fluid in participating laboratories. ARMed data indicated that methicillin-resistant Staphylococcus aureus (MRSA) was widely prevalent in the southern and eastern Mediterranean countries, with the highest proportions seen in Egypt and Malta. A significant percentage of resistance in $S$. pneumoniae was reported by several countries, whereas a worrying increase of multi-drug resistance was identified in $E$. coli, especially to third-generation cephalosporins [6].

The workshop discussed antimicrobial resistance surveillance at laboratory and national levels in the different countries that collaborated in the ARMed project. The participants also reviewed the situation at the regional level to establish the most adaptable ways in which reliable data can be achieved. Internal and external quality assurance (EQA) systems were deemed to be crucial to ensure such validation. ARMed laboratories participated in two EQA exercises in collaboration with UK-NEQAS; these exercises illustrated that, in most instances, routinely reported results have sufficient accuracy to provide good estimates of overall resistance prevalence and trends. They allowed a number of participating laboratories to identify aspects of routine culture and sensitivity that need improvement, particularly in detection of methicillin resistance in S. aureus and identification of enterococci and vancomycin susceptibility testing for this genus. It was necessary to identify future funding opportunities to maintain and expand the excellent collaboration already in place. ARMed data can be used to prioritize future areas of research.

\section{Recommendations}

- The regional surveillance effort instigated by ARMed needs to be continued. As a minimum, surveillance efforts should be retained at national levels, eventually increasing the number of participating laboratories and countries.

- Internal quality control should be undertaken at regular intervals in each participating centre.

- Diagnostic accuracy should be improved through capacity building.

- Minimum national standards for susceptibility testing should be formulated and reviewed on a yearly basis.

- Participants should work toward national reference structures.

\section{Workshop 2: Proper Antibiotic Use in Hospitals and Community}

The project assessed the health care systems in the participating countries, the availability of data on antibiotic use and expenditures in hospitals, and the 
current sources of information on antibiotic consumption data. From the information obtained, a protocol was developed for a pilot study to collect and collate data on antibiotic consumption within the Mediterranean partners, focusing mainly on systemic antibiotics used in hospital settings in the participating countries. Prospective collection of antibiotic consumption data was undertaken for a period of 24 months using a methodology based on that adopted by the ESAC project.

This workshop discussed antibiotic usage data at local and national levels in the different ARMed countries as well as region-specific issues. Extensive attention was given to the participants' individual experiences and views of the most likely ways in which valid and reliable data can be achieved in order to propose recommendations to participating countries.

Several methodological challenges were identified. Available data in the region is mostly based on hospital procurement as opposed to individual patients or wards, while a considerable volume of outpatient antibiotic use is included in the database of some hospitals. Defined daily dose (DDD) estimates do not necessarily reflect prescribed daily dose (PDD) and are not appropriate for paediatric hospitals. Additional denominators (e.g. number of admissions) need to be considered in addition to the conventional bed days; however, this information can be difficult to source. Accurate interpretation of usage patterns requires additional information on hospital characteristics and case-mix. There are several external influences on prescribing within the region, including donations that are not registered at pharmacy level, medical representatives who regularly attend most of the participating hospitals and often leave samples for the treatment of patients, and widespread personal sponsorship for congress attendance, education, and social events.

\section{Recommendations}

- An alternative and better way to obtain and evaluate antibiotic consumption data for hospitals is needed than that available to date. It may be particularly useful to develop methods that standardize a measure for longitudinal analysis of antibiotic use by hospitals that can be applied in all Mediterranean countries.

- Whole-hospital, antimicrobial usage data that is categorized by class should be recorded quarterly (yearly if based on pharmacy purchases) using the WHO defined unit of DDD per 100 patient-days and the ATC classification system. This should include:

o routine measurement of in-patient use at hospital level annually for pharmacy data; quarterly for patient data

- monitoring of $\mathrm{AB}$ use should be complemented by alternative numerators and denominators

- relevant systemic antibacterials and antifungals, their subgroups, and individual drugs (ATC5), including route of administration (oral, parenteral, rectal)

$\circ$ high/low levels and changes in consumption should be investigated and documented

- Participation in multi-centre/multinational benchmarking surveillance networks should be encouraged.

- Antimicrobial usage data should be reviewed according to individual wards/units, diagnosis, and proportion of patients treated with an antimicrobial agent.

- Antimicrobial usage data should be combined with drug utilization prevalence studies at the patient level (duration of therapy, treatment failure). This is best achieved initially through point prevalence studies.

- Antimicrobial usage data should be conveyed to prescribers.

\section{Workshop 3: Infection Prevention and Control}

ARMed evaluated infection prevention and control (IC) policies and practices through the use of structured questionnaires. Participants were asked to provide feedback on current knowledge of hospitalacquired infection (HAI) surveillance and infection control policies, personnel, and practices in their individual countries.

This workshop discussed infection control priorities at local and national levels in the different Mediterranean countries as well as other European nations to reduce the burden of hospital acquired infections (HAIs). Participants achieved a consensus within country or regional groups. The analysed data were relayed to the whole group to further facilitate discussions. Three questionnaires were completed to explore the national situation in the region that would inform the potential drivers and possible bottlenecks 
relevant to IC, as well as to identify local and national priorities. recommendations for implementation. There were mixed feelings about the effectiveness of

Table 1: Data from participating Mediterranean countries at the Consensus Meeting

on the presence of various drivers related to HAI in their respective countries

\begin{tabular}{lc}
\hline Categories & $\begin{array}{c}\text { Percentage } \\
\text { present }\end{array}$ \\
\hline IC included in the Accreditation & $33 \%$ \\
Insurance systems in place & $33 \%$ \\
Insurance premiums consider IC & $17 \%$ \\
National IC Targets published & $17 \%$ \\
High bed occupancy posing IC issues & $50 \%$ \\
Possibilities to address high bed occupancy & $17 \%$ \\
Health and Safety Executive or equivalent present & $100 \%$ \\
Audits on IC held in hospitals & $33 \%$ \\
Country has signed up to the WHO Global Patient Safety & $17 \%$ \\
Challenge & $50 \%$ \\
\hline
\end{tabular}

Hospital accreditation systems were present in half of the countries and a third of these included infection control in their assessments (Table 1). There was a Health and Safety Executive or an equivalent organization in all Mediterranean countries, but few audited any aspect of infection control. High bed occupancy posed IC issues in some of the countries, and in one of these it was thought possible to address them at the political level.

All local infection control priorities listed in Table 2 were considered important. The European countries scored audit/process surveillance and engaging the Hospital Chief Executive/Board as higher priorities than the Mediterranean countries, perhaps because they have been successful in educating staff and introducing guidelines. At the national level, the Mediterranean participants gave the highest priority to the involvement of key policy makers and politicians, followed by the assurance that IC was given importance on hospital boards. Regulation of IC at the national level (e.g. by passing national laws) was third. These top three priorities were also the top three for the other attending countries, but in reverse order. Involving health care workers in IC and establishing IC competencies were in the middle third for all countries. There were differences in the order of other IC priorities, but it was again emphasized that these were minor because all were important and should be shown to Mediterranean countries as involvement of patients in infection control and health care associated infection. It was felt that this should be separated as a priority from that of engaging and educating the media on the problem of HAIs, the socio-economic burden they comprise, and the importance of adequate resourcing of infection control (spending money to save money). It was pointed out that opportunities created by "new cards" could happen very quickly and had the potential to make a large impact, especially via the media; thus one would need to be prepared to use these opportunities to one's advantage at very short notice. This event, for example, could be the acquisition of a hospital infection by a high-profile politician, sporting or media personality.

There were many commonalities between the regions and some interesting differences in the order of the recommendations. Some of these might be explained by the Mediterranean countries' situation of being less resourced for IC (e.g. fewer have infection control teams), and health care associated infection (HCAI) control thus having a lower priority. Mediterranean participants felt that they might be able to advance more rapidly by quoting the experiences and strategies now used by many countries in the EU. Regulation was seen as a very important factor in generating the change required. The meeting showed that there were many cultural 
and political changes needed, and that background contextual differences were important in control measures, and that they realize that they must own the problem and solutions.

Table 2: Local infection control priorities categorised by workshop participants from Mediterranean and other countries. listed in descending Mediterranean countrv order.

\begin{tabular}{|c|c|c|}
\hline Categories & $\begin{array}{c}\text { Mediterranean } \\
\text { Countries }\end{array}$ & $\begin{array}{c}\text { Other } \\
\text { Countries }\end{array}$ \\
\hline Education of IC team and all health care workers & 1 & 4 \\
\hline Guidelines/policies on infection control structures and procedures & 2 & 3 \\
\hline $\begin{array}{l}\text { Use patient safety, antimicrobial stewardship as "new cards" to help } \\
\text { generate improved IC }\end{array}$ & 3 & 9 \\
\hline Interventions to prevent or control HAIs & 4 & 6 \\
\hline Engage the hospital chief executive/board in importance of IC & 5 & 2 \\
\hline $\begin{array}{l}\text { Seek healthcare worker champions for IC so that it is everyone's } \\
\text { business }\end{array}$ & 6 & 8 \\
\hline $\begin{array}{l}\text { Surveillance of infection rates, device usage and antibiotic } \\
\text { resistance \& usage }\end{array}$ & 7 & 7 \\
\hline Audit/process surveillance & 8 & 1 \\
\hline Alert organism surveillance and outbreak investigation & 9 & 5 \\
\hline Improve local laboratory practices to support IC e.g. typing & 10 & 10 \\
\hline Research and development related to infection control & 11 & 11 \\
\hline
\end{tabular}

understanding how countries viewed their priority setting.

\section{Recommendations}

The following are listed in the order they were agreed upon by the Mediterranean countries' delegates, although all were thought to be important:

- It is essential to involve all the key policy makers and politicians to ensure that HCAI and the associated issues of antibiotic resistance become and remain high national priorities.

- This approach should resonate through to ensuring that these issues are also considered as high priorities on hospital boards.

- Experience throughout the EU and Mediterranean countries indicates that improvement in HCAI and antimicrobial resistance (AMR) prevention and control requires a national regulatory strategy.

- There should be adequate funding of research and development to inform and provide the evidence required for guideline development, prevention, and control measures.

- It is vital that all the appropriate health care workers are involved in infection prevention and
- Training is essential to ensure that Infection Control teams and all health care workers have the relevant competencies in infection control.

- Patient advocates and the press/media should also be involved in the process of increasing the priority given to HCAI and AMR prevention and control.

\section{Conclusion}

The ARMed project and its concluding consensus conference have confirmed the numerous challenges posed by multi-resistant pathogens to the treatment of infectious diseases in the Mediterranean region. These problems span across many countries and involve several bacterial species, yet many of the underlying factors show significant overlap. Furthermore, the opportunity afforded by a meeting including stakeholders from the countries in the region has enabled a list of interventions and recommendations to be compiled that are both relevant and applicable to the region. These recommendations are, by necessity, not all evidencebased but have face value. Importantly, the expert 
opinions and their multi-disciplinary backgrounds at the meeting provide very significant support to their application.

It is clear that the participants showed general agreement on the need for surveillance and audit to underpin any intervention to tackle antimicrobial resistance. This applies to monitoring of changing epidemiological trends in antimicrobial susceptibilities within critical pathogens as well as identifying antibiotic consumption practices and effectiveness of prevention and control of health care associated infections, since both are critical contributory factors. In addition, the need to convey these data to key users and to improve the education and training of health care workers was also stressed in all workshops. The recommendations also clarify that ownership of the problem must be universal and that resources, both financial as well as human, must be allocated by the respective policy makers in order to combat it.

The conference consensus recommendations have been widely disseminated throughout the region, including to national health authorities as well as to individual hospitals and non-governmental organizations. It is hoped that they will serve as a catalyst to improve collaboration between the diverse stakeholders and maintain the momentum of the ARMed project in increasing our knowledge and efforts towards antibiotic resistance surveillance and control in the whole of the Mediterranean region.

\section{Acknowledgements}

Members of the ARMed Steering Group and Collaborators were: M. A. Borg (Project Leader), E. Scicluna, P. Zarb, H. Grundmann, E. Tiemersma, N. van de Sande-Bruinsma, M. de Kraker, J. Monen, H. Goossens, M. Ferech, B.D. Cookson, D. Gür, S. Ben Redjeb, O. Rasslan, Z. Elnasser, M. Benbachir, D. Pieridou Bagatzouni, K. Rahal, Z. Daoud.

\section{References}

1. Kunin CM (1993) Resistance to antimicrobial drugs - a worldwide calamity. Ann Intern Med 118: 557-61.

2. Cornaglia G, Hryniewicz W, Jarlier V, Kahlmeter G, Mittermayer H, Stratchounski L, Baquero F; ESCMID Study Group for Antimicrobial Resistance Surveillance (2004) European recommendations for antimicrobial resistance surveillance. Clin Microbiol Infect 10: 349-83.

3. Bronzwaer S, Lonnroth A, Haigh R (2004) The European Community strategy against antimicrobial resistance. Euro Surveill 9: 30-4. Available: http://www.eurosurveillance.org/em/v09n01/0901-223.asp. Accessed 28 July 2009.

4. Gur D, Unal S (2001) Resistance to antimicrobial agents in Mediterranean countries. Int J Antimicrob Agents 17: 21-26.

5. Borg MA, Scicluna E (2004) Antibiotic resistance in the Mediterranean region: the ARMed project. Eurosurveillance

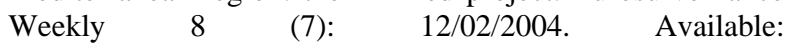
http://www.eurosurveillance.org/ew/2004/040212.asp\#5. Accessed 28 July 2009.

6. Borg MA, Scicluna E, de Kraker M, van de Sande-Bruinsma N, Tiemersma E, Gür D, Ben Redjeb S, Rasslan O, Elnassar Z, Benbachir M, Pieridou Bagatzouni D, Rahal K, Daoud Z, Grundmann H, Monen J (2006) Antibiotic resistance in the southeastern Mediterranean - preliminary results from the ARMed project. Euro Surveill 11:164-7.

\section{Corresponding Author}

Dr. Michael A. Borg

ARMed Project

Infection Control Unit

Mater Dei Hospital

Msida, Malta

Tel: $\quad$ (+356) 25451154

Fax: $\quad$ (+356) 25452140

E-mail: michael.a.borg@gov.mt

Conflict of Interest: No conflict of interest is declared 